\title{
Teaching evidence based medicine in family medicine
}

\author{
Davorka Vrdoljak
}

Department of Family Medicine University of Split, School of Medicine Split, Croatia

Corresponding author:

Davorka Vrdoljak

Doverska 23

21000 Split

Croatia

davorka.vrdoljak@mefst.hr

Tel./Fax: + 38521568696

Received: 30 November 2011

Accepted: 30 December 2011

Copyright (C) 2012 by

Academy of Sciences and Arts

of Bosnia and Herzegovina.

E-mail for permission to publish:

amabih@anubih.ba
The concept of evidence based medicine (EBM) as the integration of clinical expertise, patient values and the best evidence was introduced by David Sackett in the 1980's. Scientific literature in medicine is often marked by expansion, acummulation and quick expiration. Reading all important articles to keep in touch with relevant information is impossible. Finding the best evidence that answers a clinical question in general practice (GP) in a short time is not easy. Five useful steps are described -represented by the acronym " $5 \mathrm{~A}+\mathrm{E}$ ": as sess, ask, acquire, appraise, apply and evaluate. The habit of conducting an evidence search "on the spot" is proposed. Although students of medicine at University of Split School of Medicine are taught EBM from the first day of their study and in all courses, their experience of evidence-searching and critical appraisal of the evidence, in real time with real patient is inadequate. Teaching the final-year students the practical use of EBM in a GP's office is different and can have an important role in their professional development. It can positively impact on quality of their future work in family practice (or some other medical specialty) by acquiring this habit of constant evidence-checking to ensure that best practice becomes a mechanism for life-long learning. Conclusion. EBM is a foundation stone of every branch of medicine and important part of Family Medicine as scientific and professional discipline. To have an EB answer resulting from GP's everyday work is becoming a part of everyday practice

Key words: Evidence based medicine, Critical appraisal, Family medicine.

\section{Shift of paradigm: from "eminence" to "evidence based medicine"}

The term "Evidence Based Medicine" (EBM) has been coined by a group of clinicians and epidemiologists from McMaster University in Ontario, Canada (1). Originating from their Critical Appraisal series published in 1981 (2) the EBM model was adopted in 1992 in the Journal of American Medi- cal Association (JAMA) (3). According to David Sackett, evidence based medicine is defined as the "conscious, indisputable and critical use of the best actual evidence for decision making in care for a patient" $(1,4)$. This applies to the use of evidence in practice, in diagnostics and cure as well as in prevention and prognosis of disease. Decision making in medicine should be based on "the best evidence" resulting from research 
with the least likelihood of the many types of bias. This should start with systematic reviews of randomized controlled trials; if not available then look for individual randomized controlled trials. evidence of high quality that "can be trusted". They are based on large scale projects which gave consistent results so repeating the same ones would give similar results. Not so long ago the choice of treatments was mainly based on "established practice", "authorities' opinions" and experiences ("eminence based medicine") but that classical paradigm is nowadys replaced by EBM, with search and use of the best evidence reulting from research. Although use of the established practice is undoubtedly easier, more simple and comfortable for a doctor who is in constant search for fresh evidence, scientific progress in medicine is marked by expansion, cummulation and quick expiration, especially in propulsive fields such as neuroscience, genetics and cell and molecular biology (5-7). A medical humorist Oscar London in his book "Kill as few patients as possible" (published in 1987) has thus calculated that a general practitioner (GP) should read one review every 30 minutes in each day and night to stay in touch with information! That is impossible so we need selection in order to narrow the choice of articles which are required for a GP to optimise best- practice. A useful and simple way of finding the best evidences is to use the " 5 easy steps" (acronym $5 \mathrm{~A}+\mathrm{E}$ ) approach. The first step is to ASK and is followed by next steps: ASK, ACQUIRE; APPRAISE, APPLY and ASSESS. The clinical question is defined according to PICO principle (Patient, Intervention, Comparison and Outcome). We first have to define whom is information required for (age, gender, race), intervention type we are interested in (diagnostic test, medication, surgical procedure, advice), other possible types of intervention and their outcome (8). Second step is the choice of data basis [Co- chrane library of reviews, Medline (OVID, Pubmed)] with stress on type of study and the power of study. The third step covers the critical appraisal of answered questions: are the results of study valid, what is their nature and can they be used in the care of our individual patient. In the fourth step, the GP discusses and provides patient with the evidence on the important harms and benefits of the intevention. In the process the GP accepts the patient as a "partner in care" thus taking into consideration the personal choice, wishes, needs and qualities of patient as a whole person in his biopsychosocial, family and working surrounding. Strengthening the active role of patient in this proces in an important task of GP since the definite decision is made not by the doctor or family but by a patient himself. Patient decision aids are available on the Internet to help patients in their decision making with their GPs. Finally the doctor should evaluate how successfully these four steps have been fulfilled, are there any changes needed and what is there to do to make care for a patient better.

\section{Is it possible to use and teach EBM in Croatian family medicine setting?}

EBM includes three components: the best evidence, individual expertise and patient's choice / personal values. One can ask if it is possible to use and teach EBM in everyday work of preoccupied GP and if the answer is yes, under which preconditions? After the Project of the primary health care computerisation is conducted, all GP offices in the Republic of Croatia were informatically equiped (computers, approach to Internet) and electronic medical records were introduced as well. Technical preconditions exist in the office of each GP in Croatia so it is much easier to find information sources and search for the answer to a required question in real time, when it appears. There is a mul- 
titude of information but it is important to choose a suitable information source which best "covers" GP's needs and requirements in everyday care for patient. The source can be approached right after history taking and clinical checkup of patient, when the question is defined or it can be postponed until after the patient leaves the office with the aim to collect the information in time for the next visit. The GP rarely has the time to take a look at all original studies (primary publications) since that takes too much time. Summarized conclusions of relevant studies are much more suitable and take much less time to answer the question that has just appeared in GP's practice. With improving the habit of an evidence search "on the spot" and with more practical experience, the search takes less time In order to make information selection even more easier and more adjusted to the needs of general practice there's been adopted the idea of "simplified and concentrated EBM for Family Medicine", with acronim POEM (Patient Oriented Evidence That Matters) and more recently PEARLS (Practical Evidence About Real Life Situations) $(9,10)$. Among the most used POEMs are the ones of American Family Physician, British Medical Journal and Journal of Family Practice where groups of experts search and appraise bibliography on frequent clinical issues which are then summarized into short structured form of answers. PEARLS published by Cochrane Primary Health Field are created for primary care practitioners to help them decide in individual cases.. POEMs and PEARLS are published monthly and can be seen on web and are also sent daily on doctors' e-mail adresses upon request. They both focus on what is important from the GP's perspective as well as from patient's perspective: symptoms, morbidity, life quality, mortality. POEMS cover most common clinical problems but not all questions are analyzed (11-13).

\section{Perspectives}

Although students of medicine at University of Split School of Medicine are taught EBM from the first day of their study and in all courses, their experience of evidence searching and critical appraisal of the evidence, in real time with real patient in front of them is not large. Experiencing the partnership and holistic approach towards patient and continuous care is what they often meet for the first time in a GP's office. That is the reason why teaching the last year students the practical use of EBM in GP's office is different and can have an important role in their professional development. It can have a positive impact on quality of their further work in family practice [or some other medical specialty] as well as acquiring the habit of constant information-checking to ensure best practice and make this a lifelong learning behaviour (14-17). Sometimes for students working in family medicine during the course is the first chance to work independently "using their own hands". Then they can practice communication skills with patient as a whole person, get to know their stories, gain experience and take full responsibility. They have a chance to answer the question "What have I learned?", which is then followed by reflection and a long-term recall. The practice has shown that knowledge of the last year students is based on clinical experience of working in the hospital. Patients on which they were learning and acquiring skills are often unrepresentative of patients in the community: they often have complex problems or rare diseases and conditions not looked after in family medicine. They tend to focus on the condition and pay less attention the whole of their person. What they are introduced to in family medicine is a different approach: a GP gives continuous care for defined population through time, observes a 
person holistically, in biopsychosocial context, knowing the family and working background, taking into consideration personal values and specifities of patient as a partner in care $(18,19)$. Diseased people coming to GP are "unselected", often coming even for "small diseases" and self-limiting conditions. Specific elements of a GP's work is also a larger number of "smaller" contacts with patients, active listening, individual intervention, preventive work, long term following of patients with chronic diseases, advising and home care. All of these specific elements students of family medicine are in most cases introduced only in the final year of studying. This is an ideal time in their personal development to show them how to use EBM in family medicine and to nurture their interes in primary care. Students rate this experience very highly and accept the chance for learning how to give the best evidence to the patient who is a person with his own priorities, attitudes, preferences, family relationships, quality and dinamics of life and who is not just another "case" of aortic stenosis or depression. We can say that EBM is a foundation stone of every branch of medicine and an especially important part of family medicine as a scientific and professional discipline. Founding journal clubs for GPs who are mentors of the last year medical students could be the way to introduce and teach EBM and to spread it more widely (20). To have an EB answer on the questions raised from GP's everyday work is becoming an inevitable part of everyday practice. It is an important frame for decision making but always beginning and ending with our patient as the focus of care.

Conflict of interest: The author declare that she has no conflict of interest. This article was not sponsored by any external organisation.

\section{References}

1. Sackett DL, Rosenberg WMC, Gray JAM, Haynes RB, Richardson WS. Evidence based medicine: what it is and what it isn't? BMJ. 1996;312:71-5.

2. Department of Clinical Epidemiology and Biostatistics, McMaster University: How to read clinical journals: I. Why to read them and how to start reading them critically. Can Med Assoc J. 1981;124:555-8.

3. Evidence-Based Medicine Working Group. Evidence-Based Medicine: a new approach to teaching the practice of medicine. JAMA. 1992;268(17):2420-25.

4. Grad R, Macaulay AC, Warner M. Teaching evidence-based medical care: description and evaluation. Fam Med. 2001;33(7):602-6.

5. Alper BS, Hand JA, Elliott SG, Kinkade S, Hauan MJ, Onion DK, et al. How much effort is needed to keep up with the literature relevant for primary care. J Med Libr Associat. 2004;92(4):429-37.

6. Shaunessy AF, Slawson DC, Bennett JH. Becoming an information master: a guidebook to the medical information jungle. J Fam Pract. 1994;39:489-99.

7. Shaunessy AF, Slawson DC. Are we providing doctors with the training and tools for lifelong learning? BMJ. 1999;319:1-3

8. Smith R. What clinical information do doctors need? BMJ. 1996;313:1062-8.

9. Smith R. A POEM week for the BMJ. BMJ. 2002;325:983.

10. Hague J. Patient-oriented evidence that matters: POEMs. EJHP. 2004;2:56-7.

11. White B. Making evidence-based medicine doable in everyday practice. www.aafp.org/fpm Accessed September 2011.

12. Jones TW, West CP, Newman JP. In search of the facts: evidence-based medicine through the ages. JCOM. 2011;18(5):205-10.

13. Guyatt G, Haynes B, Jaeschke R, Cook D, Greenhalgh T, Meade M, Green L, Naylor C, Wilson M, McAlister F, Richardson M. Introduction: the philosophy of evidence-based medicine. In: Guyatt G, Rennie D, editors. Users' guides to the medical literature: a manual for evidence-based clinical practice. Chicago: AMA Press; 2002. p. 3-12.

14. Haynes BH. What kind of evidence is that Evidence-Based-Medicine adviocates want health care providers and consumers to pay attention to? BMC Health Services Research. 2002;2(3):1-7.

15. Henning G, George J. Teaching evidence-based medicine in a small rural family medicine practice office. Fam Med. 2003;35(4):241-2. 
16. Lang E. The why and the how of evidence-based medicine. MJM. 2004;8:90-4.

17. Anonymous. Evidence Based Medicine.www:// pennstatehershey.org/web/cpc/home/ebm Accesed August 2011.

18. Shuuval K, Shachak A, Linn S, Brezis M, FederBubis P, Reis S. The impact of an evidence-based medicine educational intervention on primary care physicians: a qulititative study. J Gen Intern Med. 2007;10:327-31.
19. Shawn ST, Dantas GC, Upshur REG. Evidencebased medicine in primarey care: qualitative study of family physicians. BMC Family Practice. 2003;4(6):1-9.

20. Cramer SC, Mahoney MC. Introducing evidence based medicine to the journal club, using a structured pre and post test: a cohort study. BMC Medical Education. 2001;1(6):1-4. 